\title{
Quantum computing for all?
}

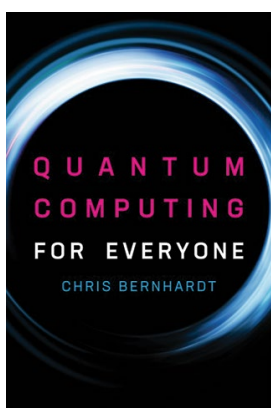

\author{
Quantum Computing \\ for Everyone
}

By Chris Bernhardt

MIT PRESS: 2019

216PP. $£ 20.00$

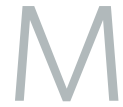

ax Planck was awarded the Nobel Prize in Physics 100 years ago for his discovery of energy quanta. At the time, notions of digital computing were somewhat premature, and it would take several decades before the foundations of modern computing systems were established. As for quantum computing, that would have to wait until almost the end of the 20th century to get going. But over the past 25 years or so, quantum computing scientists have been hard at work figuring out how to build a quantum computer and figuring out what to do with it. Today, we have a much clearer picture of both concepts, and commercial systems are even starting to appear. Chris Bernhardt's Quantum Computing for Everyone, which provides a basic introduction to the field, could have been written at any time in the past 25 years, but only now does it feel like the right time for everyone to read it.

Accounts of quantum computing abound in popular science books, magazines and even newspapers. These typically aim to provide an introduction to quantum computing for a lay audience, but the result is usually a muddled mess of bad analogies and fantastical claims: objects occupying two places at the same time, quantum computers capable of checking all solutions to a problem at once. Bernhardt intentionally avoids introducing quantum computing in a superficial way. Instead, he aims to leave the reader with a competent understanding of quantum computing, and does this assuming that they have a minimal technical background.

From the outset though, Bernhardt restricts the audience from "everyone" to those "comfortable with high-school mathematics". Yes, this book has maths in it - and not just a few equations to illustrate a point. It has calculations that you need to follow in detail - with pen and paper - to understand the point.
Nevertheless, the sophistication of the mathematics is at the level advertised, and is readily digestible by mathematically competent high-school students.

The explanatory innovation used by Bernhardt is to dispense with imaginary numbers. For a physical theory almost defined by the introduction of imaginary numbers, this is an audacious move. However, even by slicing off the imaginary part of the qubits (bits of quantum information), we can still understand the aspects of quantum physics that enable quantum computing: superposition and entanglement. Bernhardt introduces these concepts in detail using simple linear algebra.

Both superposition and entanglement rely on the concept of state. In physics, broadly speaking, the state is a complete description of a physical system from which all other quantities of interest can be derived. For example, if you know the position and momentum of a ball at any point during its flight, you can solve for its entire motion, past and future - the position and momentum are its state. While this sounds intuitive, it is important to remember that this state is, in practice, mathematical in nature. In quantum physics, the state is also stated in mathematical terms. However, unlike the classical states of a ball, when the mathematical values representing two different possible states of a quantum system are added together, the result is another possible state. It is as if these mathematical objects are being stacked together, or superimposed, and the resulting state is called a superposition. When such a superposition state is created from quite different states (qubits) of pairs of quantum systems, the result is entanglement. And when one of a pair of entangled qubits is measured, it affects the second qubit, even if they are far apart.

But here lies the problem with high-level descriptions of quantum phenomena - it is unclear how they can be useful in aiding quantum computation. And thus, Bernhardt avoids such intuitive descriptions of the phenomena and instead teaches the maths required.

After a relatively lengthy introduction to classical logic and computation, Bernhardt arrives at quantum computing, which is described at the level of detailed quantum algorithms using linear algebra and logic gates. He discusses the standard algorithms from the mid-1990s, notably leaving out the recent high-profile work on intermediatescale quantum computation. This is partly out of necessity, as contemporary research requires more sophisticated mathematics to understand, but is also in line with Bernhardt's focus on well-established ideas.

In the final chapter, Bernhardt provides an overview of the potential impact of quantum computation across many disciplines, including cryptography, big data, chemistry, optimization problems and theoretical computer science. Again, this is kept brief as the author sensibly abstains from making predictions about future technological advances.

Quantum Computing for Everyone is pure exposition. While it seems that Bernhardt has no central thesis to put forward, the implicit argument is this: to really understand quantum computing you need to understand the mathematical language in which it is written. This point makes the book stand apart from others. It is not quite a technical textbook and not quite a popular science book, it exists somewhere in between - there is probably a quantum superposition joke in that.

On my bookshelf sits my own book, Quantum Computing for Babies, next to the canonical textbook on the topic, Quantum Computation and Quantum Information by Michael Nielsen and Isaac Chuang. One is for infants and the other is for graduate students. Quantum Computing for Everyone will fit nicely between the two.

As someone who often entertains students interested in quantum computing, the book is, for me, a turning point. My heart sinks when a prospective student excitedly tells me they are interested in the discipline because they read a popular science book that paints quantum physics as an exciting place of parallel universes full of open questions ready for the next young hotshot to make their seminal contribution. Quantum Computing for Everyone is a much-needed dose of reality, and an honest path for the earnest beginner.

\section{Reviewed by Chris Ferrie \\ Centre for Quantum Software and Information, University of Technology Sydney, Sydney, New South Wales, Australia. \\ e-mail:christopher.ferrie@uts.edu.au}

Published online: 15 March 2019

https://doi.org/10.1038/s41928-019-0223-4 